\title{
Is the Conjunction Fallacy tied to Probabilistic Confirmation?
}

\begin{abstract}
Crupi, Fitelson, and Tentori (CFT, forthcoming) offer three conditions that constitute an attempted confirmation-theoretic explanation of the conjunction fallacy. I present experimental results to show that CFT's conditions are neither necessary nor sufficient for the fallacy. There exist cases that do not meet their three conditions in which subjects still tend to commit the fallacy. Also, there exist cases that meet all three of CFT's conditions in which subjects do not tend to commit the fallacy. In light of these experiments, CFT's account of the conjunction fallacy cannot be right as it stands.
\end{abstract}

Word count: 4,360

\section{Introduction - Probability, Confirmation, and the Conjunction Fallacy}

In 'Probability, Confirmation, and the Conjunction Fallacy,' Vincenzo Crupi, Branden Fitelson, and Katya Tentori (CFT) offer an explanation of the conjunction fallacy that appeals to Bayesian accounts of confirmation. As they write, 'a unified account of probabilistic fallacious judgments in classical conjunction problems could be found on the basis of the notion of confirmation: participants may in fact have a tendency to rely on assessments of confirmation when judging probabilities' (p. 6). ${ }^{1}$ CFT are clear that

\footnotetext{
Thanks very much to Jason Byron, David Danks, Jonathan Livengood, Justin Sytsma, and especially to Edouard Machery for help and advice on this project.

Address for correspondence:

Department of History \& Philosophy of Science

University of Pittsburgh

1017 Cathedral of Learning

Pittsburgh, PA 15260

USA

Email: jns24@pitt.edu
} 
their account does not excuse humans from the charge that they tend to commit a genuine fallacy; experimental subjects do make 'a genuine error given the experimental task' ( $\mathrm{p}$. 6). However, they suggest that 'the fallacious probability judgments experimentally observed are typically guided by sound assessments of confirmation relations, meant in terms of contemporary Bayesian confirmation theory' (p. 1).

Bayesian confirmation theory has proposed a host of probabilistic measures of the confirmatory support given to an hypothesis $h$ by some evidence $e$. All such measures are functions of a probability distribution over statements $h$ and $e$ that output a unique real number. At minimum, all Bayesian measures agree that this real number is positive in the case that $p(h \mid e)>p(h)$, negative in the case that $p(h \mid e)<p(h)$, and zero whenever $p(h \mid e)=p(h)$. Table 1 summarizes the various Bayesian measures of confirmation explicitly discussed by CFT.

Table 1. Bayesian Measures of Confirmation

$\begin{array}{ll}D(h, e)=p(h \mid e)-p(h) & \text { (Carnap, 1950; Eells, 1982) } \\ R(h, e)=\ln \left[\frac{p(h \mid e)}{p(h)}\right] & \text { (Keynes, 1921; Milne, 1996) } \\ L(h, e)=\ln \left[\frac{p(e \mid h)}{p(e \mid \neg h)}\right] & \text { (Good, 1950; Fitelson, 2001) } \\ C(h, e)=p(h \& e)-p(h) \times p(e) & \text { (Carnap, 1950) } \\ S(h, e)=p(h \mid e)-p(h \mid \neg e) & \text { (Christensen, 1999; Joyce, 1999) } \\ Z(h, e)= \begin{cases}\frac{p(h \mid e)-p(h)}{1-p(h)} \text { if } p(h \mid e) \geq p(h) \\ \frac{p(h \mid e)-p(h)}{p(h)} \text { otherwise }\end{cases} & \text { (Crupi, Tentori \& Gonzalez, 2007) }\end{array}$

\footnotetext{
${ }^{1}$ All pages numbers refer to the online pre-print found at $\langle$ http://fitelson.org/pccf.pdf $\rangle$.
} 
Whenever a formal result pertaining to Bayesian confirmation holds regardless of the measure one chooses, it is said to be robust. ${ }^{2}$ Here, I follow CFT in using the symbol $c(h, e)$ to denote generically any of the Bayesian measures of confirmation listed in Table 1.

The conjunction fallacy occurs when subjects rank a conjunction $h_{1} \& h_{2}$ more probable than one of its conjuncts $h_{1}$ in light of some information or evidence $e$; that is, contra the axioms of probability theory, subjects affirm that $p\left(h_{1} \& h_{2} \mid e\right)>p\left(h_{1} \mid e\right){ }^{3} \mathrm{CFT}$ offer both a general thesis and a specific thesis pertaining to the conjunction fallacy in terms of Bayesian confirmation theory. CFT's general thesis is that people 'may depart from the relevant probabilistic relationship between $p\left(h_{1} \& h_{2} \mid e\right)$ and $p\left(h_{1} \mid e\right)$ because of the perception that $c\left(h_{1} \& h_{2}, e\right)>c\left(h_{1}, e\right)^{\prime}$ (p. 6). Their specific thesis is that the following three conditions are tied to the conjunction fallacy:

(i) $\quad e$ is negatively (if at all) correlated with $h_{l}$ [i.e., $c\left(h_{1}, e\right) \leq 0$ ];

(ii) $\quad e$ is positively correlated with $h_{2}$, even conditionally on $h_{1}$ [i.e., $c\left(h_{2}, e\right)>0$ and $\left.c\left(h_{2}, e \mid h_{1}\right)>0\right]$

(iii) $\quad h_{1}$ and $h_{2}$ are mildly (if at all) negatively correlated [i.e., $c\left(h_{1}, h_{2}\right) \leq 0$ ]. As CFT note, conditions (i) and (ii) robustly imply that $c\left(h_{1} \& h_{2}, e\right)>c\left(h_{1}, e\right)-$ the relevant confirmation relation that their general thesis requires for the conjunction fallacy. ${ }^{4}$ Thus, if an experimental case meets conditions (i) and (ii), the relevant confirmation relation will be present regardless of which Bayesian confirmation measure one chooses; in such cases, CFT posit that subjects will tend to commit the conjunction fallacy. Accordingly,

\footnotetext{
${ }^{2}$ This term is thus being used in the sense of Fitelson, 1999.

${ }^{3}$ For the most well-known, early account of the conjunction fallacy, see Tversky and Kahneman, 1983.

${ }^{4}$ See proof in CFT's appendix.
} 
CFT seem to intend their conditions as at least sufficient for the fallacy. It is less clear whether CFT intend their conditions also to be necessary for the conjunction fallacy. In part two of this paper, I will present experimental evidence to show that CFT are better off not making this claim. While this evidence does support the claim that CFT's specific conditions are positively correlated with the conjunction fallacy, it also reveals that there exist ways of getting the conjunction fallacy apart from meeting CFT's specific conditions. Thus, I first argue that while it may be the case that conditions (i) through (iii) are sufficient, they are not necessary for the fallacy.

CFT's account is not without its predecessors. ${ }^{5}$ All of these have in common that they tie the conjunction fallacy to Bayesian confirmation - usually to a specific Bayesian confirmation measure. According to these accounts (CFT's included of course), people tend to make the conjunction fallacy when the conjunction $h_{1} \& h_{2}$ is better confirmed by the evidence $e$ than the first conjunct $h_{1}$ by itself. ${ }^{6}$ In part three, I will present some experimental evidence that tells against all such confirmation-theoretic accounts of the conjunction fallacy. As I will show, the confirmation relation is insufficient for the conjunction fallacy. Thus, regarding CFT's specific confirmation-theoretic account, I show here that their conditions (i) through (iii) are not sufficient for getting the fallacy. In part four, I conclude with a brief discussion of a possible response that one could make to the work presented in this paper.

Toward the end of their article, CFT write, 'A limitation of the present account is, of course, that we are not presenting new empirical evidence in favour of it. We do think,

\footnotetext{
${ }^{5}$ CFT's survey of such predecessors includes the following: R. Hertwig and V. M. Chase, 1998; D. A. Lagnado and D. R. Shanks, 2002; I. Levi, 2004; and A. Sides, D. Osherson, N. Bonini, and R. Viale, 2002.

${ }^{6}$ CFT's specific thesis is unique insofar as, unlike their predecessors's theses, it is robust across all confirmation measures. So long as their conditions (i) and (ii) are met by a particular probability distribution, any confirmation measure will output the inequality $c\left(h_{l} \& h_{2}, e\right)>c\left(h_{1}, e\right)$.
} 
however, that relevant experimental inquiries can be devised' (p. 12). The purpose of this paper then is to put forth some new empirical evidence pertaining to CFT's thesis. Counter to CFT's hopes, this evidence will show that their thesis cannot be right as it stands.

\section{The Conjunction Fallacy apart from CFT's Conditions}

While CFT successfully show that their conditions imply the confirmation relation $c\left(h_{1} \& h_{2}, e\right)>c\left(h_{1}, e\right)$, it is interesting to note that there exist other ways to achieve this inequality apart from meeting all three of their conditions. Trivially, this can be shown to be true given that - as CFT readily note - conditions (i) and (ii) on their own imply the inequality. Additionally, (i) and (ii) do not imply condition (iii). Accordingly, one can easily imagine cases where conditions (i) and (ii) are met [i.e., $c\left(h_{1}, e\right) \leq 0, c\left(h_{2}, e\right)>0$, and $c\left(h_{2}, e \mid h_{1}\right)>0$ ] while at the same time condition (iii) is broken [i.e., $c\left(h_{1}, h_{2}\right)>0$ ]. For example, the following seems to constitute just such a case:

e: John is walking along in the woods when he spots a black animal $h_{1}$ : The animal that John spots has feathers $h_{2}:$ The animal that John spots is a raven

In this case then, the conjunction $h_{1} \& h_{2}$ is confirmed by $e$ to a greater extent than $h_{1}$ alone is confirmed by $e$, although CFT's condition (iii) is not met.

There exist more interesting ways that one can achieve the confirmation relation $c\left(h_{1} \& h_{2}, e\right)>c\left(h_{1}, e\right)$ robustly apart form CFT's conditions. In fact, this inequality can be present in cases that either don't meet condition (i) or don't meet condition (ii). In the 
latter case, although condition (ii) is broken - and thus $e$ is either negatively correlated with $h_{2}$ if at all conditionally or unconditionally on $h_{1}$ (or both) - the confirmation relation may still be attained if $h_{l}$ is negatively correlated with $e$ to a large enough degree. ${ }^{7}$ In the former case, although $e$ is positively correlated with $h_{1}-$ and thus condition (i) is not met - it may still be the case that, given a very strong positive correlation between $e$ and $h_{2}$, the extent to which the conjunction $h_{1} \& h_{2}$ is confirmed by $e$ is robustly greater than the extent to which $h_{l}$ alone is confirmed by $e$. In such situations, it might even be the case that $h_{1}$ and $h_{2}$ are positively correlated - and thus condition (iii) is not met - and the confirmatory inequality will still follow robustly. ${ }^{8}$ This possibility raises the interesting question of whether one could get the conjunction fallacy to arise in such cases. Are CFT's specific conditions necessary for the conjunction fallacy, or are they at best merely sufficient?

In order to answer this question, I ran an experiment using the following setup: e: Linda participates in anti-war protests, votes Democrat, and subscribes to a popular liberal magazine.

$h_{1}$ : Linda is a poet $h_{2}:$ Linda is a feminist

${ }^{7}$ For example, any probability distribution specifying the following assignments:

$$
p\left(h_{1}\right)=.5, p\left(h_{2}\right)=.2, p(e)=.4, p\left(h_{1} \& h_{2}\right)=.1, p\left(h_{1} \mid e\right)=.1, p\left(h_{2} \mid e\right)=.2, p\left(h_{2} \mid h_{1}\right)=.2, p\left(h_{1} \& h_{2} \mid e\right)=.05
$$

will robustly lead to the result that $c\left(h_{1} \& h_{2}, e\right)>c\left(h_{1}, e\right)$ in spite of the fact that condition (ii) is broken (given that $h_{2}$ is not positively correlated with $e$ given that $\left.p\left(h_{2}\right)=p\left(h_{2} \mid e\right)\right)$.

${ }^{8}$ For example, any probability distribution specifying the following assignments:

$$
p\left(h_{1}\right)=.1, p\left(h_{2}\right)=.2, p(e)=.05, p\left(h_{1} \& h_{2}\right)=.05, p\left(h_{1} \mid e\right)=.5, p\left(h_{2} \mid h_{1}\right)=.5, p\left(h_{1} \& h_{2} \mid e\right)=.48
$$

will robustly lead to the result that $c\left(h_{1} \& h_{2}, e\right)>c\left(h_{1}, e\right)$ in spite of the fact that conditions (i) and (iii) are broken (given that $h_{l}$ is positively correlated with $e$ and $h_{l}$ and $h_{2}$ are positively correlated). 
Test subjects $(N=56$; age: mean $=19.8$, standard deviation $=1.76 ; 41.4 \%$ male $)$ were all drawn from undergraduate courses offered through the Department of History \& Philosophy of Science at the University of Pittsburgh. Through a series of 'Background Information' questions, it was determined that no subjects had any significant formal mathematical training. When asked which of two statements was more likely to be true in light of information $e$, test subjects were forced to choose between '(a) Linda is a poet' and '(b) Linda is a feminist poet.' The order of answer choices was randomized to eliminate any potential ordering effects. In order to gauge test subjects' degrees of belief pertaining to CFT's three conditions, I asked subjects three questions (see appendix 1 for all test questions).

My predictions for this experiment were as follows: For the majority of subjects, CFT's conditions would not be met given that condition (i) could plausibly be taken to be broken given a judged positive correlation between $e$ and $h_{l}$ and given that condition (iii) could also plausibly be taken to be broken given a judged positive correlation between $h_{l}$ and $h_{2}$. Most importantly, I predicted that, in spite of the fact that subjects would tend to believe that CFT's conditions are not met in this case, many subjects would still commit the conjunction fallacy.

These predictions were borne out in the experimental results: $56.9 \%$ of subjects explicitly expressed belief that the case breaks with CFT's conditions in one way or another. Out of these 32 subjects who expressed such belief, $37.5 \%$ still committed the conjunction fallacy. On the other hand, out of the 24 subjects who expressed the belief that the probe meets CFT's conditions, 70.8\% committed the fallacy (see Figure 1). 


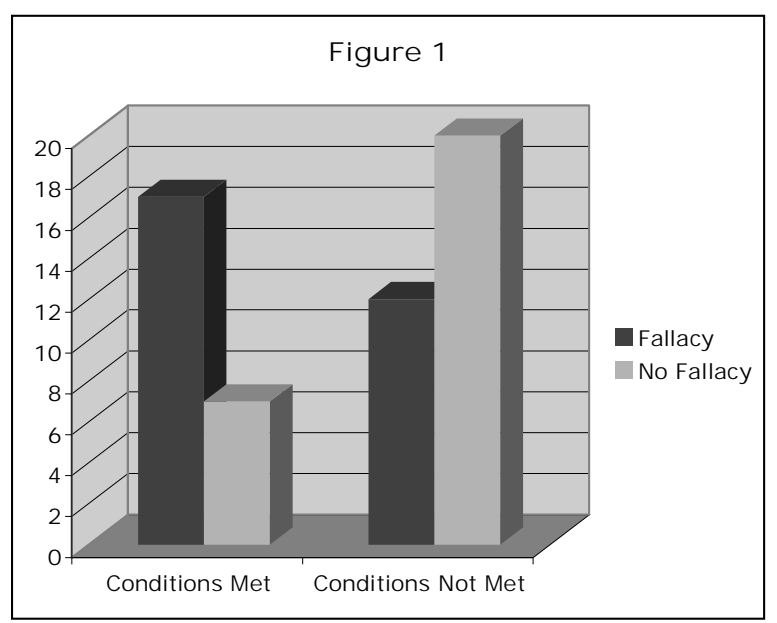

These results show us at least two things: first, we see that there exists a clear significant difference between those subjects who believe that the case meets CFT's conditions and those who do not $\left(\chi^{2}(d f=1, N=56)=6.1 ; p<.05\right)$. The former group has a stronger tendency to commit the fallacy then the latter. This supports the thesis that CFT's conditions are positively correlated with the conjunction fallacy.

Secondly however, these results show that there exist cases in which subjects still tend to commit the conjunction fallacy in spite of the fact that they believe one or more of CFT's conditions are not met by the case. While it's true that subjects who believed that CFT's conditions were met by this case were far more likely to make the fallacy then those who didn't, nearly $40 \%$ of subjects who explicitly expressed belief that this case broke with CFT's conditions made the conjunction fallacy nonetheless. Thus, this experiment constitutes strong evidence that CFT's conditions, though clearly positively correlated with the fallacy, are not necessary for getting subjects to commit the conjunction fallacy. 


\section{CFT's Conditions apart from the Conjunction Fallacy}

It's not clear whether CFT would have any problem with my results as presented in part two. Although the experimental results in part two imply that their conditions are not necessary for the conjunction fallacy, CFT could rightly respond that they show this simply by exploiting alternative ways that one can get the relevant, general confirmation relation apart from their specific conditions. Insofar as this confirmation relation is still

present, these results only add support to their general thesis that the conjunction fallacy is tied to probabilistic confirmation. Pertaining to their specific thesis, CFT could argue that they never intended their conditions to be necessary but only sufficient for the fallacy. In this part then, I show that CFT's conditions - and the presence of the relevant Bayesian confirmation relation more generally - also are not sufficient for the conjunction fallacy.

One interesting feature of Bayesian confirmation theory is that it pays little heed to the prior probabilities of hypotheses per se when deciding whether these are or aren't confirmed by some information. This is of course an attractive feature of such accounts; it seems appropriate to affirm that an hypothesis either is or isn't confirmed by evidence regardless of how probable that hypothesis is apart from the evidence. To be more precise, it is only once one compares posteriors to priors in some way that priors have some say in whether or not a hypothesis is confirmed in the Bayesian sense; i.e., it seems right that priors should only matter to confirmation relative to posteriors (and vice versa, as CFT point out: posteriors should only - it would seem - have some say in confirmation relative to priors). Given this feature of probabilistic confirmation, it is 
quite possible for the confirmation relation $c\left(h_{1} \& h_{2}, e\right)>c\left(h_{1}, e\right)$ to hold even in cases where $p\left(h_{1}\right)>>p\left(h_{1} \& h_{2}\right)$ and $p\left(h_{1} \mid e\right)>>p\left(h_{1} \& h_{2} \mid e\right)$. This leads to the following interesting question: in such cases, will subjects still make the conjunction fallacy?

In order to answer this question, I ran two additional experiments. The setup was identical to the first experiment. Subjects were again drawn from undergraduate courses offered through the Department of History \& Philosophy of Science at the University of Pittsburgh (subjects did not overlap between the first and second sets of experiments). Through a series of 'Background Information' questions, it was determined that few to no subjects had any significant formal mathematical training. These experiments were run with a between-subjects design, and the order of answer choices was randomized to eliminate any ordering effect. In both cases, I asked subjects three questions to gauge their degrees of belief pertaining to CFT's three conditions (see appendices 2 and 3).

In the first experiment (appendix 2), subjects ( $N=20$; age: mean=19.8, standard deviation $=.90 ; 10.0 \%$ male) were faced with the following setup:

e: Jenny is planning on trying out for American Idol this upcoming season $h_{1}$ : Jenny's favorite music is opera $h_{2}$ : Jenny will be the next American Idol

When asked which of two statements was more likely to be true in light of information $e$, subjects were forced to make a choice between 'Jenny's favorite music is opera' and 'Jenny's favorite music is opera and she will be the next American Idol.'

In the second experiment (appendix 3), subjects $(N=21$; age: mean=20.6, standard deviation $=2.23 ; 23.8 \%$ male) were faced with the following setup: e: Jim is an occasional purchaser of a mega millions lottery ticket 
$h_{1}$ : Jim is a scientist

$h_{2}$ : Jim will win the multi-million dollar jackpot in the lottery

When asked which of two statements was more likely to be true in light of information $e$, subjects were forced to make a choice between 'Jim is a scientist' and 'Jim is a scientist and he will win the multi-million dollar jackpot in the lottery.'

My predictions for both of these experiments were as follows: In both cases, subjects would tend to agree that CFT's conditions are met - if $h_{l}$ (being a scientist) would be seen as relevant at all either to $e$ (being an occasional lottery player) or to $h_{2}$ (being a lottery winner), it would be seen as negatively so; also, subjects would tend to see a positive correlation between being an occasional lottery player and being a lottery winner. Most importantly, I predicted that, in spite of this fact, subjects would not tend to make the conjunction fallacy in these cases.

Again, all of my predictions were borne out by the experimental results. Pertaining to the 'American Idol' case, $75 \%$ of subjects explicitly agreed that CFT's conditions were met. Out of these subjects who expressed such belief, $80 \%$ did not commit the fallacy (see Figure 2). Additionally, in the 'Lottery' case, $66 \%$ of subjects agreed that CFT's conditions were met. Out of these subjects, $85.7 \%$ did not commit the fallacy (see Figure 3). 

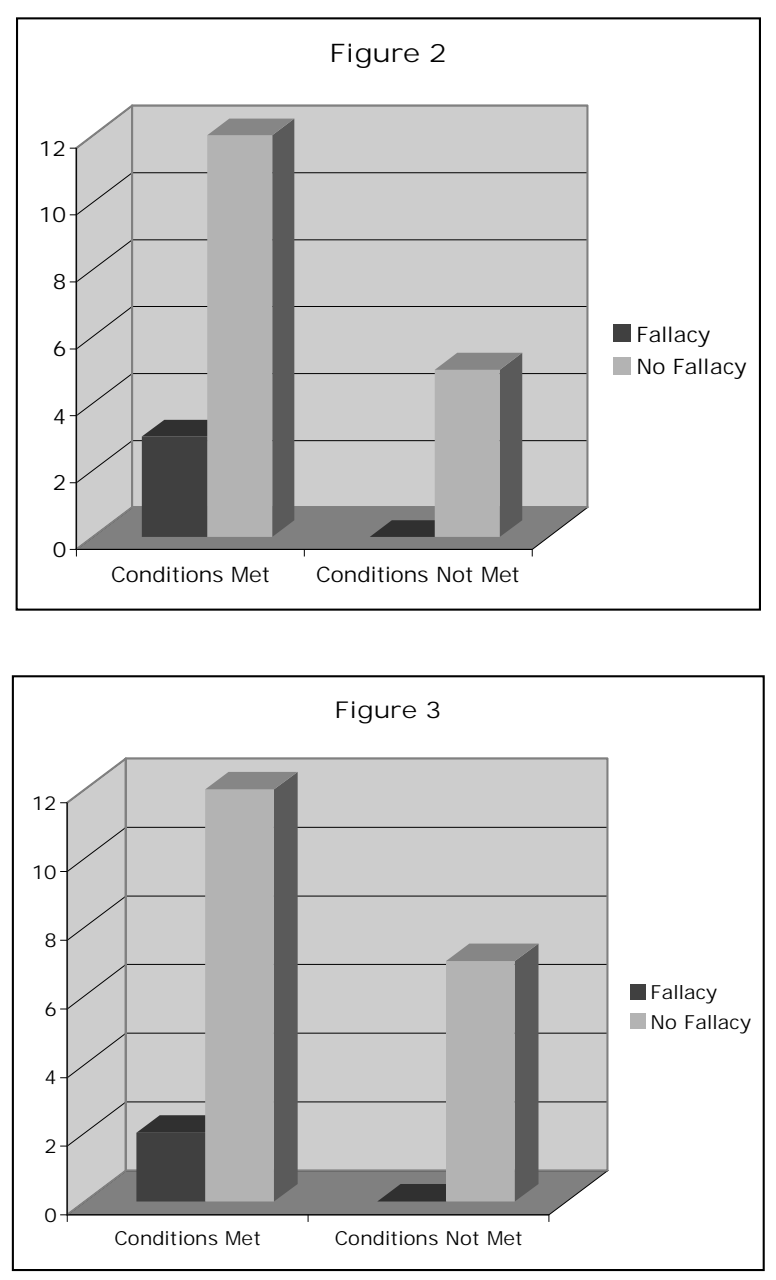

These results show that there exist cases in which subjects do not tend to commit the fallacy in spite of the fact that they believe that all three of CFT's conditions are met. Thus, these experiments constitute strong evidence that CFT's conditions are not sufficient for getting subjects to commit the conjunction fallacy.

It is especially important here to remind ourselves of the robustness of CFT's thesis in terms of their specific three conditions. As noted above, CFT prove that their conditions (i) and (ii) imply the confirmation relation $c\left(h_{1} \& h_{2}, e\right)>c\left(h_{1}, e\right)$ robustly - i.e., regardless of what specific measure of confirmation one chooses. Thus, the consequence of these two experiments can be critically applied to any Bayesian confirmation-theoretic account of the conjunction fallacy whatever. That is, CFT as well as all of their 
confirmation-theoretic predecessors (see footnote 8) may be criticized on the charge that their accounts specify conditions for the conjunction fallacy that are not sufficient.

\section{Conclusion}

The first experiment presented in this paper revealed that CFT's conditions are not necessary for the conjunction fallacy. The next two experiments showed that all Bayesian confirmation-theoretic accounts of the conjunction fallacy (CFT's included of course) specify conditions that are not sufficient for the conjunction fallacy. While the former result is relatively weak insofar as it is merely a critique of CFT's specific thesis (and only insofar as they actually intend their conditions to be necessary for the fallacy), the latter result has important and wide implications for many recent accounts of the conjunction fallacy. As such, it's worth considering the following reply to the latter results.

One may respond to this critique by admitting that while probabilistic confirmation per se does indeed appear insufficient for the conjunction fallacy in light of these results, it is at least a step in the right direction. Such a person might want to continue down this road then by adding some condition(s) to the relevant, general confirmation-theoretic condition and claiming that the conjunction of these are sufficient for the fallacy. Given my initial discussion in part three, it would seem that an initial attempt might include some extra condition pertaining to the prior probability of $h_{2}$;

presumably such an extra condition would need to bar cases in which a person might not 
be inclined to make the fallacy simply because the second conjunct - though confirmed by the evidence - is highly improbable.

However, my guess is that any such attempt will meet with insuperable difficulty. I am skeptical that there exists any general probabilistic threshold below which subjects will always tend not to make the fallacy and above which subjects will always tend to make the fallacy (assuming of course that the confirmation condition is met). Of course, there are additional ways that one could try to incorporate information about the prior probability of $h_{2}$ into the conditions for getting a conjunction fallacy other than via some specific probabilistic threshold. However, prima facie, it would seem that the more complex such an additional condition becomes, the less descriptive it will be of what is going on in people's heads when they commit the fallacy.

My experiments are by no means intended to be the last word on this subject; on the contrary, they are intended to be a first response to CFT's charge that their account should be checked empirically. If this paper is able to motivate additional experiments in the field (even if these do happen to show that mine were ultimately misguided) I would consider this project a complete success.

Jonah N. Schupbach

History \& Philosophy of Science

University of Pittsburgh 


\section{References}

Carnap, R. 1950: Logical Foundations of Probability. Chicago: University of Chicago Press.

Crupi, V., Fitelson, B., and Tentori, K. Forthcoming: Probability, confirmation, and the conjunction fallacy. Thinking and Reasoning.

Crupi, V., Tentori, K., and Gonzalez, M. 2007: On Bayesian measures of evidential support: theoretical and empirical issues. Philosophy of Science, 74, 299-252.

Christensen, D. 1999: measuring confirmation. Journal of Philosophy, 96, 437-461.

Eells, E. 1982: Rational Decision and Causality. Cambridge, UK: Cambridge University Press.

Fitelson, B. 1999: The plurality of Bayesian measures of confirmation and the problem of measure sensitivity. Philosophy of Science, 66, S362-S378.

---. 2001: A Bayesian account of independent evidence with applications. Philosophy of Science, 68, S123-S140.

Good, I. J. 1950: Probability and the Weighing of Evidence. London: Griffin.

Hertwig, R., and Chase, V. M. 1998: Many reasons or just one. Thinking \& Reasoning, 4, 319-352.

Joyce, J. 1999: The Foundations of Causal Decision Theory. Cambridge, UK: Cambridge University Press.

Keynes, J. M. 1921: A Treatise on Probability. London: Macmillan.

Lagnado, D. A., and Shanks, D. R. 2002: Probability judgment in hierarchical learning. Cognition, 83, 81-112.

Levi, I. 2004: Jaako Hintikka. Synthese, 140, 37-41. 
Milne, P. 1996: $\log [p(h \mid e b) / p(h \mid b)]$ is the one true measure of confirmation. Philosophy of Science, 63, 21-26.

Sides, A., Osherson, D., Bonini, N., and Viale, R. 2002: On the reality of the conjunction fallacy. Memory \& Cognition, 30, 191-198.

Tentori, K., Crupi, V., Bonini, N., and Osherson, D. 2007: Comparison of confirmation measures. Cognition, 103, 107-119.

Tversky, A. and Kahneman, D. 1983: Extensional versus intuitive reasoning: the conjunction fallacy in probability judgment. Psychological Review, 90, 293-315. 
Appendix 1. Experimental Questions - Feminist Poet

Linda participates in anti-war protests, votes Democrat, and subscribes to a popular liberal magazine

In light of this information, which statement is more likely to be true?

(a) Linda is a poet

(b) Linda is a feminist poet

separate page

1. How does learning all of this information about Linda (that Linda participates in antiwar protests, votes Democrat, and subscribes to a popular liberal magazine) affect the likelihood that Linda is a poet?

(a) This information makes it more likely that Linda is a poet

(b) This information makes it less likely that Linda is a poet

(c) This information doesn't affect the likelihood that Linda is a poet

2. How does learning all of this information about Linda (that Linda participates in antiwar protests, votes Democrat, and subscribes to a popular liberal magazine) affect the likelihood that Linda is a feminist?

(a) This information makes it more likely that Linda is a feminist

(b) This information makes it less likely that Linda is a feminist

(c) This information doesn't affect the likelihood that Linda is a feminist

3. Now consider a different person, Sue. All that you learn about Sue is that she is a poet. How does learning this information (that Sue is a poet) affect the likelihood that Sue is a feminist?

(a) This information makes it more likely that Sue is a feminist

(b) This information makes it less likely that Sue is a feminist

(c) This information doesn't affect the likelihood that Sue is a feminist 
Appendix 2. Experimental Questions - American Idol

Jenny is planning on trying out for American Idol this upcoming season

In light of this information, which statement is more likely to be true?

(a) Jenny's favorite music is opera

(b) Jenny's favorite music is opera and she will be the next American Idol separate page

1. How does learning that Jenny is planning on trying out for American Idol this upcoming season affect the likelihood that Jenny's favorite music is opera?

(a) This information makes it more likely that Jenny's favorite music is opera

(b) This information makes it less likely that Jenny's favorite music is opera

(c) This information doesn't affect the likelihood that Jenny's favorite music is opera 2. How does learning that Jenny is planning on trying out for American Idol this upcoming season affect the likelihood that she will be the next American Idol?

(a) This information makes it more likely that Jenny will be the next American Idol

(b) This information makes it less likely that Jenny will be the next American Idol

(c) This information doesn't affect the likelihood that Jenny will be the next American Idol

3. Now consider a different person, Sandy. All that you learn about Sandy is that her favorite music is opera. How does learning this information (that Sandy's favorite music is opera) affect the likelihood that Sandy will be the next American Idol?

(a) This information makes it more likely that Sandy will be the next American Idol

(b) This information makes it less likely that Sandy will be the next American Idol

(c) This information doesn't affect the likelihood that Sandy will be the next American Idol 
Appendix 3. Experimental Questions - Lottery

Jim is an occasional purchaser of a mega millions lottery ticket

In light of this information, which statement is more likely to be true?

(a) Jim is a scientist

(b) Jim is a scientist and he will win the multi million dollar jackpot in the lottery separate page

1. How does learning that Jim is an occasional purchaser of a mega millions lottery ticket affect the likelihood that Jim is a scientist?

(a) This information makes it more likely that Jim is a scientist

(b) This information makes it less likely that Jim is a scientist

(c) This information doesn't affect the likelihood that Jim is a scientist

2. How does learning that Jim is an occasional purchaser of a mega millions lottery ticket affect the likelihood that Jim will win the lottery?

(a) This information makes it more likely that Jim will win the lottery

(b) This information makes it less likely that Jim will win the lottery

(c) This information doesn't affect the likelihood that Jim will win the lottery

3. Now consider a different person, Bill. All that you learn about Bill is that he is a scientist. How does learning this information (that Bill is a scientist) affect the likelihood that Bill will win the lottery?

(a) This information makes it more likely that Bill will win the lottery

(b) This information makes it less likely that Bill will win the lottery

(c) This information doesn't affect the likelihood that Bill will win the lottery 